\title{
Distribution of the Mitochondria-Associated ER Membrane in Rat Hepatocyte Revealed by FIB/SEM Tomography
}

\author{
Keisuke Ohta ${ }^{1}$, Ryuhei Higashi ${ }^{2}$, Akinobu Togo ${ }^{2}$, Satoko Okayama ${ }^{2}$ and Kei-ichiro Nakamura ${ }^{1}$ \\ 1. Department of Anatomy, Kurume University school of Medicine, Kurume, Fukuoka, Japan \\ 2. Center Unit of Electron Microscopy, Kurume University, Kurume, Fukuoka, Japan
}

Great attention has recently been paid to the interaction between the endoplasmic reticulum (ER) and mitochondria. A physical interaction between the ER and mitochondria is referred to as the mitochondria-associated ER membrane (MAM)[1]. This physical interaction or contact has key roles in several cellular functions, including $\mathrm{Ca}^{2+}$ signaling, lipid transport, energy metabolism and apoptosis. Although the structures of the MAM have been directly visualized using conventional electron microscopic study and more modern method such as the electron tomography[2], it has been difficult to observe the entire distribution of the ER-mitochondrial contact sites on a mitochondrion. In this study, we revealed the entire three dimensional membrane organization of a $6 \mu \mathrm{m}$ cuboidal region of rat hepatocyte by serial block face imaging using focused ion beam/scanning electron microscope (FIB/SEM) in order to visualize the distribution of the ER-mitochondrial contact sites on an entire mitochondrion.

3D reconstruction method using $\mathrm{FIB} / \mathrm{SEM}$ tomography are powerful tools for understanding the mesoscale architecture of cells and tissues as it provides more detailed 3D information than the serial sectioning method of TEM, and also provides a larger volume of information than the electron tomography method [3].

The specimens were obtained from rat (Wistar, 6W) after transcardial perfusion of $2 \%$ paraformaldehyde, $2.5 \%$ glutaraldehyde and $2 \mathrm{mM} \mathrm{CaCl}_{2}$ in $0.1 \mathrm{M}$ cacodylate buffer $(\mathrm{pH} 7.4)$. After initial perfusion, the specimens were cut into small cubes about $1 \mathrm{~mm}^{3}$ and were then postfixed and en bloc stained using a combination of the ferrocyanide-reduced osmium method and the osmiumthiocarbohydrazide-osmium method to enhance membrane contrast and embedded in epoxy resin[4,5].

Serial image data set of hepatocyte for 3D reconstruction were obtained from serial cycles of ion-beam milling and compositional contrast imaging of the newly created flat surface of the block in a beam deceleration field using FIB/SEM machinery (Quanta 3D FEG, FEI Co.). Briefly, the specimens were set in a specially designed holder and the serial images were acquired under the following conditions. The milling was performed with a gallium ion beam at $30 \mathrm{kV}$ with a current of $100 \mathrm{pA}$. The milling pitch was set to $10 \mathrm{~nm} / \mathrm{step}$. Compositional contrast images were acquired at a landing energy of $3 \mathrm{keV}$ with a bias voltage of $1.5 \mathrm{kV}$ using a vCD detector [5]. The other acquisition parameters were as follows: Beam current $=11 \mathrm{pA}$, dwell time $=30 \mu \mathrm{s} /$ pixel, image size $=5.9 \mu \mathrm{m} \times 5.1 \mu \mathrm{m}$, pixel size $=5.8 \mathrm{~nm} / \mathrm{pixel}$. The cycle was repeated 650 times. The resultant image stack was processed using Avizo 6.3 software (VSG Inc.). Membrane components of the mitochondrion including its cristae structure were segmented by semi-automatically manners.

The final reconstructed volume clearly depicted the ER contact sites on the outer mitochondrial membrane (OMM) that showed two types of membrane interaction. One is a tether structure between ER and mitochondria that has been reported in mammalian cells. This tethering structure resembles a 
protein complex structure that is referred to as ERMES (ER-mitochondria encounter structure) that has been identified in yeast[6]. The other is direct adhering between the ER membrane and OMM. These two types of MAM are existed on a mitochondrion simultaneously, but the distribution of these structures seems to form clusters of the same type of associations on the OMM independently. These results imply functional differences of the two types of MAM on a mitochondrion.

References:

[1] T Hayashi, R Rizzuto, G Hajnoczky and TP Su, Trends Cell Biol 19 (2009), p. 81.

[2] G Csordás et al, J Cell Biol 174 (2006), p. 915-921.

[3] G Knott, H Marchman, D Wall, B Lich, J Neurosci 28(2008), p. 2959.

[4] G Perkins, C Renken, ME Martone, SJ Young, M Ellisman, T Frey, J Struct Biol, 119 (1997), p. 260.

[5] K Ohta, S Sadayama, A Togo, R Higashi, R Tanoue, K Nakamura, Micron 43 (2012), p. 612.

[6] B Kornmann, P Walter, J Cell Sci 123 (2010), p.1389

[7] This work was supported by JSPS KAKENHI Grant Number 24590265.

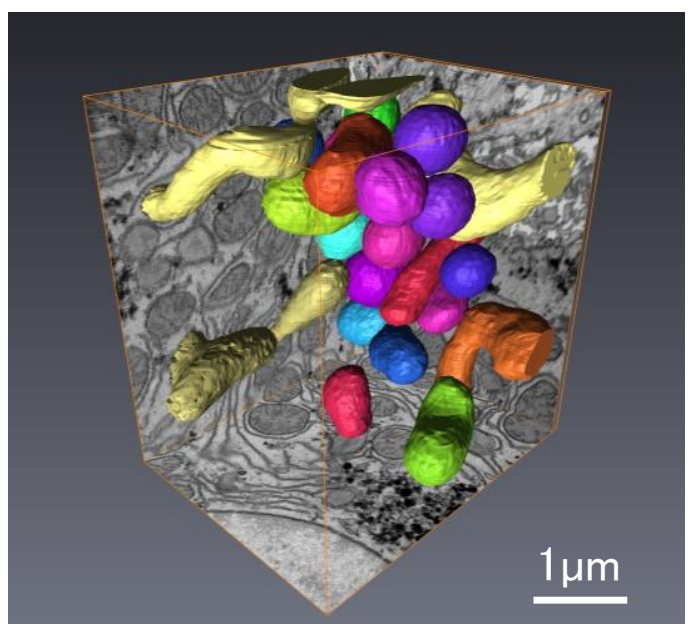

Figure 1. Mitochondria in the entire volume of the reconstructed hepatocyte cytoplasm using FIB/SEM

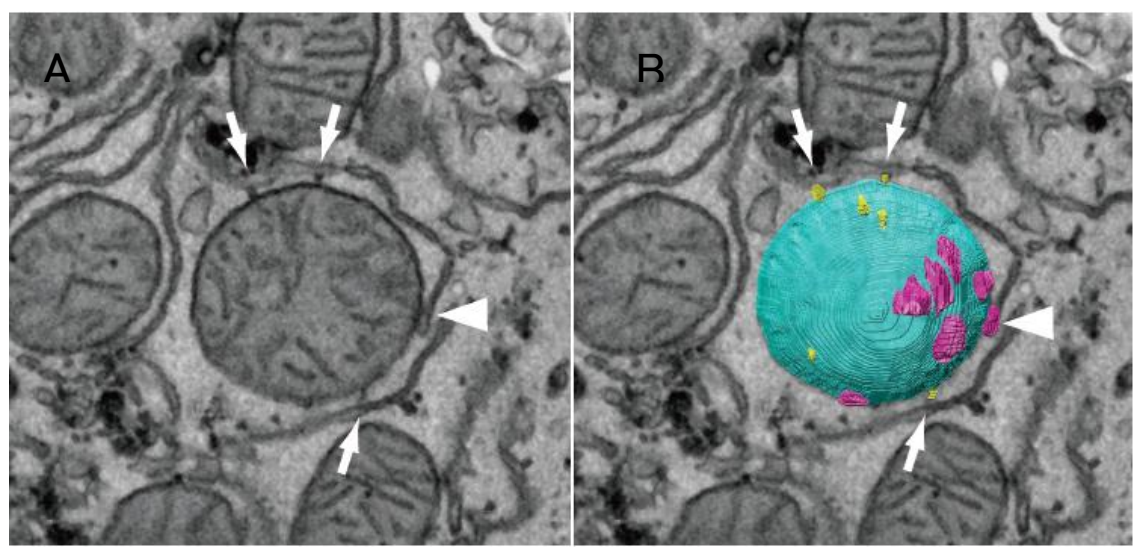

Figure 2. Distribution of the MAM structure on the outer membrane of mitochondorion (OMM)

A. A right angle slice of mitochondria of rat hepatocyte obtained using FIB/SEM. Tether structures (Arrows) and membrane adhesion (Arrowhead) on the OMM are indicated. B. A 3D model of the mitochondrion shown in A and the distribution of MAMs. (Tether structures shown in yellow, membrane adhesions shown in purple.) 